\title{
Screening for inherited thrombophilias and prophylaxis of venous thromboembolism in pregnancy and puerperium
}

\author{
Ana SCUTELNICU ${ }^{1}$, Corina GICA ${ }^{1}$, Radu BOTEZATU ${ }^{1,2}$, Nicolae GICA ${ }^{1,2}$, \\ Anca Marina CIOBANU ${ }^{1}$, Brindusa $\mathrm{CIMPOCA}^{1}$, Gheorghe PELTECU ${ }^{1,2}$, \\ Anca Maria PANAITESCU ${ }^{1,2}$ \\ ${ }^{1}$ Filantropia Clinical Hospital, Bucharest, Romania \\ 2"Carol Davila" University of Medicine and Pharmacy, Bucharest, Romania
}

\begin{abstract}
Thrombophilias are a group of coagulation disorders associated with a predisposition to thrombotic events. They could be inherited (genetic) or acquired. The most encountered inherited thrombophilias are factor $V$ Leiden (FVL), prothrombin gene mutation (G20210A) (PGM), protein S deficiency, protein C deficiency and antithrombin (AT) deficiency. Based upon the risk of thrombosis, there are two types of inherited thrombophilias, high risk and low risk. The high risk inherited thrombophilia includes antithrombin III deficiency, factor $V$ Leiden homozygotes, prothrombin gene mutation and compound heterozygotes FVL and PGM. The low risk thrombophilia includes factor $V$ Leiden heterozygote, PGM heterozygote, protein C deficiency and protein $S$ deficiency. The incidence of all thromboembolic events is estimated to approximately 1-2 per 1000 pregnancies and about an equal number are identified antepartum and in the puerperium. Screening for thrombophilia in pregnancy is not universally recommended because of the low incidence of the condition and because it is not cost-effective. Some adverse pregnancy outcomes could be associated with some types of thrombophilia, but this association is weak and there is no reason to initiate extensive investigations or therapeutic measures. Both obstetricians and hematologists and women need to be correctly informed and avoid emotional decisions and unnecessary invasive treatments.
\end{abstract}

Keywords: pregnancy, puerperium, inherited thrombophilia, venous thromboembolism, prophylaxis

\section{INTRODUCTION}

Pregnancy is a procoagulant state, probably acquired during evolution, to ensure hemostasis during normal pregnancy and particularly during delivery, when a certain blood loss is expected, being threatening to pregnant woman's life. Because venous thromboembolism (VTE), term which includes both deep vein thrombosis and pulmonary embolism, is an important cause of morbidity and mortality during pregnancy and the post-partum period, it is essential for all clinicians to know how to prevent it, when and which patients will benefit from prophylaxis and if the thrombotic risk will surpass the bleeding risk.

Thrombophilias are a group of coagulation disorders associated with a predisposition to thrombotic events. They could be inherited (genetic) or acquired. The most encountered inherited thrombophilias are factor $V$ Leiden $(F V L)$, prothrombin gene mutation (G20210A) (PGM), protein S deficiency, protein C deficiency and antithrombin (AT) deficiency. Antiphospholipid antibody syndrome is considered an acquired 
thrombophilia and is diagnosed based on clinical history and laboratory testing [1]. Based upon the risk of thrombosis, there are two types of inherited thrombophilias, high risk and low risk. The high risk inherited thrombophilia includes antithrombin III deficiency, factor $\mathrm{V}$ Leiden homozygotes, prothrombin gene mutation and compound heterozygotes FVL and PGM. The low risk thrombophilia includes factor $\mathrm{V}$ Leiden heterozygote, $\mathrm{PGM}$ heterozygote, protein $\mathrm{C}$ deficiency and protein $\mathrm{S}$ deficiency [1].

The incidence of all thromboembolic events is estimated to approximately $1-2$ per 1,000 pregnancies and about an equal number are identified antepartum and in the puerperium. In a study from Norway of more than 600,000 pregnancies, Jacobsen et al. [2] reported that deep-vein thrombosis alone was more frequent antepartum, whereas pulmonary embolism was more common in the first 6 weeks postpartum. Specifically, Berg et al. [3] reported that approximately $10 \%$ of pregnancy related maternal deaths in the United States between 1998 and 2005 were caused by thrombotic pulmonary embolism.

The overall maternal death incidence in the USA, related to pulmonary embolism, is estimated to 1,2 at 100,000 deliveries [4]. It is estimated that the incidence of deep vein thrombosis is much higher, accounting for 71 at 100,000 deliveries and factor V Leiden (FVL) is involved in $8 \%$ of the cases. Based on these data, the thrombotic risk for a woman during pregnancy and puerperium is estimated at 1 in 400 to 500 [5]. If $50 \%$ of the cases of lethal pulmonary embolism would occur in the $10 \%$ of women with $\mathrm{FVL}$, the risk would be 1 in 13,000 .

Factor V Leiden and prothrombin G20210A (also called the prothrombin gene mutation -PGM) together account for 50 to 60 percent of cases of an inherited (primary) hypercoagulation state in Caucasian populations. Deficiencies in protein S (PS), protein C (PC), and antithrombin (AT) account for most of the remaining cases [1].

\section{ETIOLOGY AND PATHOPHYSIOLOGY}

Changes in the coagulation system, an increase of venous stasis, vascular injury at delivery and hypercoagulability (Virchow's triad) substantially increase the risk of developing VTE in pregnancy compared to the non-pregnant state. Changes in the coagulation system during pregnancy include: increases in fibrinogen and factors II, VII, VIII, IX, X and XII; an increased activity of the fibrinolytic inhibitors as evidenced by increases in plasminogen-activator inhibitor 1 (PAI-1) and 2 (PAI-2); a decrease in protein S (PS) activity; and an increase in resistance to activated protein $C$ in the second and third trimesters. In approximately $50 \%$ of patients with a hereditary thrombophilia, the initial thrombotic event occurs in the presence of an additional risk factor such as pregnancy, personal or family history, high body mass index, smoking, oral contraceptive use, orthopedic trauma, immobilization or surgery [6].

\section{INHERITED OR ACQUIRED THROMBOPHILIA AND PREVIOUS VTE}

They are the most important risk factors that increase the prothrombotic obstetrical state, which is already six times higher during the third trimester and 22 times higher during the first 6 weeks postpartum compared to non-pregnant women, according to Sultan et al. [7]. Most discrepancies in the current guidelines are related to prophylaxis in women with no prior VTE. Although evidence-based national and international guidelines recommendations for the risk assessment and thromboprophylaxis for this patient population have been published, they are largely based upon observational studies and extrapolated from data of non-pregnant patients. The lack of high quality data, specific to pregnancy, results in a lack of consistency in their recommendations, especially in women with no personal history of thrombotic events.

Differences in a VTE risk threshold will lead to different recommendations for prophylaxis during pregnancy. For example, the Society of Obstetricians and Gynecologists of Canada (SOGC) and the American College of Chest Physicians (ACCP) provide different recommendations for postpartum prophylaxis, based on different VTE thresholds ( $1 \%$ and $3 \%$, respectively). Based on an anonymous vote of international thrombosis pregnancy experts, the majority $(60 \%)$ have chosen $3 \%$ or higher as a VTE risk threshold for antepartum prophylaxis and $3 \%$ or higher as a VTE risk threshold for postpartum prophylaxis; however, not all experts agreed. Acknowledging that risk thresholds differ between guidelines and clinicians is an important aspect of managing pregnant women at risk [8].

There are many inhibitory proteins that regulate the coagulation cascade. Inherited or acquired deficiencies of these inhibitory proteins are collectively referred to as thrombophilia. These can lead to hypercoagulability and recurrent VTE. Although these disorders are collectively present in approximately $15 \%$ of white European populations, they are responsible for approximately $50 \%$ of all thromboembolic events during pregnancy [6]. The most common inherited defect is the factor $V$ Leiden, heterozygote mutation (1-15\%), also known as activated protein $\mathrm{C}$ resistance, as presented in Table 1. 
TABLE 1. Inherited thrombophilia and their association with VTE in pregnancy (adapted after the American College of Obstetricians and Gynecologists, 2013).

\begin{tabular}{|l|c|c|c|c|}
\hline & $\begin{array}{c}\text { Prevalence } \\
(\%)\end{array}$ & $\begin{array}{c}\text { VTE risk per } \\
\text { pregnancy } \\
(\%) \\
\text { H- }\end{array}$ & $\begin{array}{c}\text { VTE risk per } \\
\text { pregnancy } \\
(\%) \\
\text { H+ }\end{array}$ & $\begin{array}{c}\text { All VTE } \\
\text { (\%) }\end{array}$ \\
\hline FVL heteroz. & $1-15$ & $0.5-1.2$ & 10 & 40 \\
\hline FVL homoz. & $<1$ & 4 & 17 & 2 \\
\hline PT heteroz. & $2-5$ & $<0.5$ & $>10$ & 17 \\
\hline PT homoz. & $<1$ & $2-4$ & $>17$ & 0.5 \\
\hline $\begin{array}{l}\text { FVL and PT } \\
\text { heteroz. }\end{array}$ & 0.01 & $4-5$ & $>20$ & $1-3$ \\
\hline $\begin{array}{l}\text { PC activity } \\
\text { (<50\%) }\end{array}$ & $0.2-0.4$ & $0.1-0.8$ & $4-17$ & 14 \\
\hline PS (<55\%) & $0.03 .-01.3$ & 0.1 & $0-22$ & 3 \\
\hline
\end{tabular}

H - history; VTE - venous thromboembolism; FVL - factor V Leiden; PT - prothrombin20210A gene mutation; PC - protein C; PS - protein S free antigen

An estimated 20 to $50 \%$ of women who develop a venous thrombosis during pregnancy or postpartum have an identifiable underlying genetic disorder [9]. But because the overall absolute thrombotic risk is still low during pregnancy, current guidelines do not recommend general screening for thrombophilia.

\section{SCREENING FOR INHERITED THROMBOPHILIA}

There are confusing data related to the screening of pregnant women for inherited or acquired thrombophilia, especially because of the cost-benefit matter. As mentioned before, all current guidelines agree that the absolute incidence of VTE during pregnancy is low, about 1-2 cases per 1000 pregnancies. Considering that the pregnant women represent about $5 \%$ of the population at any given time (according to World Health Organization), one can truly perceive the scale of the problem. Nevertheless, all expert organizations agree that universal screening is not a viable option for most health care systems, so a selective screening strategy is required.

The level of VTE risk during pregnancy depends upon whether the pregnant woman has a high- or lowrisk thrombophilia and whether she has a personal or family history of VTE [1].

The American College of Obstetricians and Gynecologists (ACOG) [9] recommends that targeted assessment for inherited thrombophilia may be considered in case of a personal history of VTE, with or without a recurrent risk factor, and no prior thrombophilia testing, or in case of family history (a first-degree relative - parent or sibling) with a history of high-risk inherited thrombophilia. In the setting of recurrent pregnancy loss (4 miscarriages) or stillbirth, testing for the acquired antiphospholipid syndrome should be considered.
The Royal College of Obstetricians and Gynaecologists (RCOG) [10] recommends testing for antithrombin deficiency if family history of VTE and either antithrombin deficiency or no specific thrombophilia is detected. It also recommends testing for antiphospholipid antibodies if unprovoked VTE.

It is recommended to consider thrombophilia testing, even in the absence of personal history or risk factors for VTE, in the presence of history of unprovoked or estrogen-provoked VTE in first-degree relative, when aged $<50$ years; better to have more information if a relative has known thrombophilia.

The Society of Obstetricians and Gynaecologists of Canada (SOGC) [11] does not recommend routine screening for all inherited thrombophilia in women with a first episode of VTE diagnosed in pregnancy. Testing for protein $\mathrm{S}$, protein $\mathrm{C}$ and antithrombin deficiencies is indicated following a venous thromboembolism in pregnancy if there is a family history of these particular thrombophilia or if thrombosis occurs in an unusual site. Testing for antiphospholipid antibodies is indicated only if the results would affect the duration of anticoagulation.

According to Maternal-Fetal Evidence Based Guidelines (2017) [6], it is recommended to screen any pregnant woman with a current or prior personal history of VTE, as this could affect anticoagulation recommendations, especially if the event was not associated with a clear precipitating event, such as immobilization after surgery. They argue that there is insufficient evidence to support thrombophilia screening in case of family history.

All expert organizations agree that screening should include factor $\mathrm{V}$ Leiden-homozygote, prothrombin 20210A gene mutation-homozygote, antithrombin III deficiency, protein $S$ deficiency and protein $C$ deficiency. Protein C and PS assessment is less reliable during pregnancy. Screening for methylenetetrahydrofolate reductase (MTHFR) and, plasminogen activator inhibitor-1 (PAI-1) are not recommended, although many laboratories include them in their thrombophilia panel, probably for the financial reasons.

According to Middeldorp S, a leading expert in the field of thrombophilia, testing for the inherited thrombophilic defects is a "double-edged sword" [12]. Firstly, testing is costly and diagnostic tests without clinical utility should not be performed. Secondly, because of the psychological and social effects of knowing that one is a carrier, with no proved clinical implication. Thirdly, because of the pitfalls of laboratory testing and a potential false reassurance of non-carriers who have an increased VTE risk based on family history alone, probably because of another genetic mutation not yet discovered. There are all reasons to be more restrictive in recommending testing. 
The current guidelines agree on a selective screening strategy, even though there are some minor differences in recommendations. The most important risk indicators for VTE during pregnancy, that should imply screening for thrombophilia, are personal history of VTE or family history of VTE (as unprovoked or estrogen provoked VTE in a first-degree relative, when aged is $<50$ years), with/without known thrombophilia mutation. Venous thromboembolism is a multifactorial disease caused by hereditary and acquired risk factors. Thrombosis occurs more often in the first-degree relatives (parents, siblings, children) of patients with VTE, than in the general population, even in the absence of a known thrombophilia [13-15], probably because there is another hereditary component involved, yet to be discovered.

\section{INDICATIONS FOR PROPHYLAXIS}

The decision to provide thromboprophylaxis to pregnant women is based on the following factors: any prior personal history of VTE; the presence of high- versus low-risk thrombophilia; the setting in which a prior VTE occurred (pregnancy/estrogen-related, idiopathic, or in the setting of non-recurrent risk factors such as fractures, surgery, or immobilization). The decision to provide thromboprophylaxis is accompanied by the decision of timing (antenatal or postpartum or both) and regimen [16]. Early recognition of risk factors and adequate implementation of the thromboprophylaxis guidelines will significantly reduce maternal mortality due to thromboembolism.

Current guidelines agree that a thorough prenatal risk evaluation for VTE should be performed on the first visit to the obstetrician specialist and repeated throughout pregnancy any time the clinical status changes (eg. in case of admission to hospital for medical or surgical conditions). For high-risk thrombophilia, most recommendations are for antepartum and 6-weeks postpartum thromboprophylaxis, with minor differences shown in Table 2.

It is important to underline the fact that there are not many relevant randomized control studies and most recommendations are mainly based on expert opinion rather than high-quality evidence.

\section{DISCUSSIONS}

There is a false perception of pregnant women and even of some members of the medical community that thrombophilia is responsible for many adverse pregnancy outcomes (pregnancy loss, fetal death in utero, intrauterine growth restriction, abruptio placentae) and that the wide use of thromboprophylaxis could prevent these events. Both doctors (obstetricians and hematologists) and women need to be correctly informed and avoid emotional decisions and unnecessary invasive treatments. Based on the current level of knowledge, thrombophilia seems to add a weak contribution to these adverse pregnancy outcomes.

Women with high-risk thrombophilias have a high risk of pregnancy associated VTE. In one study, the risk was found to be independent of a positive family history of thrombosis [17]. The absolute risks of thrombosis during pregnancy and the puerperium were estimated as follow: for AT deficiency, $6.1 \%$ at age $<35$ and $9 \%$ at age $\geq 35$; for FVL homozygote, $2.2 \%$ at age $<35$ and $3.4 \%$ at age $\geq 35$; for compound heterozygote $\mathrm{FVL}$ and

TABLE 2. Current guidelines recommendations for high-risk thrombophilia-adapted after (6-8)

\begin{tabular}{|l|l|l|l|}
\hline & ACOG (2018) & RCOG (2015) & SOGC (2014) \\
\hline High risk thrombophilia & FVLand PT homozygote & FVL and PT homozygote \\
& FVL + PT heterozygote & $\begin{array}{l}\text { FVL + PT heterozygote } \\
\text { AT III deficiency } \\
\text { PPS }\end{array}$ & $\begin{array}{l}\text { FVL and PT homozygote } \\
\text { FVL + PT heterozygote } \\
\text { AT III de } \\
\text { APS }\end{array}$ \\
\hline $\begin{array}{l}\text { Homozygosity FVL /PT } \\
\text { Antepartum/postpartum }\end{array}$ & Prophylaxis / Prophylaxis & $\begin{array}{l}\text { Consider prophylaxis/ } \\
\text { Prophylaxis 6w }\end{array}$ & Prophylaxis / Prophylaxis \\
\hline $\begin{array}{l}\text { Heterozygosity FV and PT } \\
\text { Antepartum/postpartum }\end{array}$ & Prophylaxis / Prophylaxis & $\begin{array}{l}\text { Consider prophylaxis/ } \\
\text { Prophylaxis 6w }\end{array}$ & Prophylaxis / Prophylaxis \\
\hline $\begin{array}{l}\text { AT III deficiency } \\
\text { Antepartum/postpartum }\end{array}$ & Prophylaxis / Prophylaxis & $\begin{array}{l}\text { Consider prophylaxis/ } \\
\text { Prophylaxis 6w }\end{array}$ & Prophylaxis / Prophylaxis \\
\hline $\begin{array}{l}\text { Protein C or S deficiency } \\
\text { Antepartum/postpartum }\end{array}$ & $\begin{array}{l}\text { Surveillance or prophylaxis/ } \\
\text { Surveillance or prophylaxis if RF }\end{array}$ & $\begin{array}{l}\text { Consider prophylaxis/ } \\
\text { Prophylaxis 6w }\end{array}$ & $\begin{array}{l}\text { Surveillance / surveillance or } \\
\text { prophylaxis 6w if >=1RF }>1 \%\end{array}$ \\
\hline $\begin{array}{l}\text { APS } \\
\text { Antepartum/postpartum }\end{array}$ & $\begin{array}{l}\text { Surveillance or prophylaxis / } \\
\text { prophylaxis }\end{array}$ & $\begin{array}{l}\text { Prophylaxis if >=3RF or from } \\
28 w \text { if >= 2RF / } \\
\text { Prophylaxis 6w }\end{array}$ & $\begin{array}{l}\text { Intermediate or ther } \\
\text { prophylaxis / Prophylaxis 6w }\end{array}$ \\
\hline
\end{tabular}

ACOG - American College of Obstetricians and Gynecologists; RCOG - Royal College of Obstetricians and Gynecologists; SOGC - Society of Obstetricians and Gynecologists of Canada; FVL - factor V Leiden; PT - prothrombin20210A gene mutation; AT III - antithrombin III deficiency; APS - antiphospholipid syndrome; RF-risk factors; $w$ - weeks 
prothrombin gene mutation (PGM), 5.5\% at age 35 and $8.2 \%$ at age $\geq 35$ [17].

Screening for thrombophilia in pregnancy is not universally recommended because of the low incidence of the condition and because it is not cost-effective [18].

Screening for inherited thrombophilia would be considered in case of personal history of venous thromboembolism (VTE) [with or without recurrent risk factors: recurrent risk factors (pregnancy or oral contraceptive users) or non-recurrent risk factors (surgery or immobilization)] or positive family history (first degree relative with history of high risk inherited thrombophilia).

Screening is not recommended in case of personal history of recurrent or non-recurrent fetal loss, preeclampsia, placental abruption or intrauterine growth restriction or preeclampsia.

When thrombophilia screening in pregnancy is considered, the panel will include FVL-homozygote, prothrombin gene mutation-homozygote, AT deficiency (activity $<60 \%$ ), protein $\mathrm{S}$ deficiency (activity $<55 \%$ ), protein $\mathrm{C}$ deficiency (activity $<65 \%$ ), anti-phospholipid antibodies (acquired thrombophilia).

\section{CONCLUSIONS}

Inherited thrombophilias are genetic disordes that expose to higher risk of thromboembolic complications during pregnancy because gestation is a procoagulant state. Some adverse pregnancy outcomes could be associated with some types of thrombophilia, but this association is weak to consider it a causality and to initiate extensive investigations or therapeutic measures. The prediction of VTE during pregnancy in women with inherited thrombophilias is based upon a specific type of thrombophilia, associated with a positive history of personal or family history of thrombosis. Screening is not recommended for high homocysteine levels, presence of MTHFR variants or PAI-1 variants. If a pregnant woman had these biochemical or genetic testing and findings are abnormal, she will be counseled that the result is of no clinical significance, she needs not thromboprophylaxis and her relatives should not be tested. Screening for thrombophilias will be recommended if it is anticipated that it will influence the management decision. The aim of thromboprophylaxis is prevention of maternal VTE. Based upon the data cited from the literature, as well as evidence from studies in nonpregnant individuals, we recommend, as the most reasonable, the approach that described in the Table 2. Hippocrates' adage, first do not harm, is still valid in case of the screening, diagnosis and prophylaxis of trombophilias.

\section{Acknowledgement}

All authors equally contributed to this article.

Conflict of interest: none declared Financial support: none declared

\section{REFERENCES}

1. Lockhood CL, Bauer KA. Inherited thrombophilia in pregnancy. UpToDate, 31 March 2020.

2. Jacobsen AF, Skjeldstad FE, Sandset PM. Incidence and risk patterns of venous thromboembolism in pregnancy and puerperium-a register-based case control study. Am J Obstet Gynecol, 2008; 198:233.el.

3. Berg CJ, Callaghan WM, Syverson C, et al. Pregnancy-related mortality in the United States, 1998 to 2005. Obstet Gynecol. 2010; 116:1302.

4. Bauer KA. Screning for inherited thrombophilia in asymptomatic adults. UpToDate, August 14, 2019.

5. McCall MD, Ramsay JE, Tait RC. Risk factors for pregnancy associated venous thromboembolism. Thromb Haemost. 1997; 78(4):1183.

6. Berghella V. Maternal-Fetal Evidence Based Guidelines, 3rd ed., 2017:261.

7. Sultan AA, West J, Tata LJ, Fleming KM, Nelson-Piercy C, Grainge MJ. Risk of first venous thromboembolism in and around pregnancy: a population-based cohort study. Br J Haematol. 2012;156(3):366-73.

8. Skeith L. Preventing venous thromboembolism during pregnancy and postpartum: crossing the threshold. Hematology Am Soc Hematol Educ Program. 2017;2017(1):160-167.

9. American College of Obstetricians and Gynecologists' Committee on Practice Bulletins - Obstetrics. ACOG Practice Bulletin No. 197: Inherited Thrombophilias in Pregnancy. Obstet Gynecol. 2018 Jul; 132(1):e18-e34.

10. RCOG Green-top Guideline, No. 37a, 2015. Thrombosis and Embolism during Pregnancy and the Puerperium, Reducing the Risk.

11. Chan WS, Rey E, Kent NE; VTE in Pregnancy Guideline Working Group, Chan WS, Kent NE, Rey E, Corbett T, David M, Douglas MJ, Gibson PS, Magee L, Rodger M, Smith RE; Society of Obstetricians and Gynecologists of Canada. Venous thromboembolism and antithrombotic therapy in pregnancy. J Obstet Gynaecol Can. 2014 Jun;36(6):527-53.

12. Middeldorp S. Inherited thrombophilia: a double-edged sword. Hematology Am Soc Hematol Educ Program. 2016 Dec 2; 2016(1):1-9.

13. Lussana F, de Rooij SR, Veenendaal M, et al. Prevalence of factor $\mathrm{V}$ Leiden and G20210A prothrombin mutation in the Dutch
Famine Birth Cohort: a possible survival advantage? Thromb Haemost. 2012; 108(2):399-401.

14. Sørensen HT, Riis AH, Diaz LJ, Andersen EW, Baron JA, Andersen PK. Familial risk of venous thromboembolism: a nationwide cohort study. J Thromb Haemost. 2011; $9(2): 320-324$.

15. Couturaud F, Leroyer C, Julian JA, et al. Factors that predict risk of thrombosis in relatives of patients with unprovoked venous thromboembolism. Chest. 2009; 136(6):1537-1545.

16. Springel EH. Thrombophilia in pregnancy. UpToDate. June 14, 2018.

17. Gerhardt A, Scharf RE, Greer IA, Zotz RB. Hereditary risk factors of thrombophilia and probability of venous thromboembolism during pregnancy and the puerperium. Blood. 2016;128(19):2343-2349.

18. Clark P, Twaddle S, Walker S, et al. Cost-effectiveness of screening for the factor $\mathrm{V}$ Leiden mutation in pregnant women. Lancet. 2002;359(9321):1919. 